\title{
Dignity Therapy Improves Hope and Quality of Life in Cancer Patients: A Randomized Clinical Trial
}

\author{
Zahra Karimi $^{1 \text { (D) , Nasim Mehrpooya }}{ }^{2}$, Seyyed Abolfazl Vagharseyyedin ${ }^{1}$ (D), Hossein Rahimi*1 ${ }^{*}$
}

1. Dept. of Nursing, School of Nursing and Midwifery, Birjand University of Medical Sciences, Birjand, Iran

2. Dept. of Nursing, School of Nursing and Midwifery, Neyshaboor University of Medical Sciences, Neyshaboor, Iran

\begin{tabular}{|c|}
\hline Article Info \\
\hline do) $10.30699 /$ jambs.28.128.156 \\
\hline $\begin{array}{c}\text { Received: 2020/04/09; } \\
\text { Accepted: 2020/07/01; } \\
\text { Published Online: 08 Jul 2020; }\end{array}$ \\
\hline $\begin{array}{l}\text { Use your device to scan and read the } \\
\text { article online }\end{array}$ \\
\hline 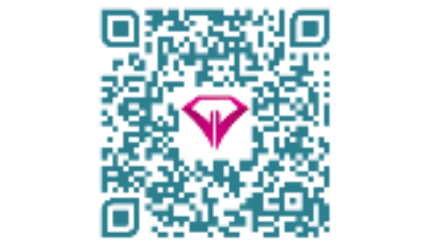 \\
\hline
\end{tabular}

Corresponding Information: Hossein Rahimi

Dept. of Nursing, School of Nursing and Midwifery, Birjand University of Medical Sciences, Birjand, Iran E-Mail:

hosseinrahimi92@yahoo.com

\section{ABSTRACT}

Background \& Objective: Cancer is one of the most prominent public health issues. It can put the patient's hope and quality of life (QOL) at risk. The purpose of this study was to determine the effect of dignity therapy on the hope and quality of life of cancer patients.

Materials \& Methods: This trial was conducted in 2019. The sample included 76 cancer patients who were randomly divided into an experimental group $(n=38)$ and a control group $(n=38)$. Data were collected using a demographic questionnaire, the Herth-Hope questionnaire (1991), and the EORTC QLQ-C30 scoring (1988). The intervention group received a dignity therapy protocol. The control group received no intervention. The questionnaires were completed again four weeks after the intervention. Data were analyzed by SPSS 16 and several tests (Chi-square, Kolmogorov-Smirnov, independent t-test, paired t-test, Wilcoxon, and MannWhitney). The significance level was set at $\mathrm{P}$-value $<0.05$.

Results: Mean scores for hope and quality of life before the intervention were not significantly different between the experimental and control groups $(P=0.11)$. Four weeks after the end of the intervention, the mean scores of hope in the intervention group (26.88 \pm 2.90$)$ were significantly higher than those of the control group ( 24.60 $\pm 4.26)(P=0.03)$. Also, after the intervention, the mean scores of quality of life in the intervention group $(69.61 \pm 12.71)$ were significantly higher than those of the control group $(50.64 \pm 12.15)(P<0.001)$.

Conclusion: Dignity therapy can be an effective intervention method for increasing hope and improving the quality of life among cancer patients.

Keywords: Cancer, Dignity therapy, Hope, Quality of life

\section{Introduction}

Cancer is one of the most prominent public health issues. In 2019, nearly 1.8 million people in the United States had cancer. It will cause approximately $70 \%$ of all deaths by 2030 (1). Although precise statistics on the prevalence of cancer in Iran are not available, Mousavi et al. (2009) have estimated the prevalence of cancer in Iran to be 98 to 110 per 100,000 individuals (2).

Cancer imposes physical, mental, and psychological complications directly and indirectly on patients. For example, headaches, dyspnea, digestive problems, and defecation disorders are some of the direct complications of cancer (3). Indirect complications are often due to the type of treatment received. For example, chemotherapy commonly leads to bone marrow suppression, skin problems, hair loss, fatigue, nausea and vomiting, neuropathy, and cardiopulmonary toxicity (4). Cancer also causes mental and psychological problems in patients. Depression, stress disorders, cognitive impairments, sexual disorders, and death anxiety are the most common psychosocial complications associated with cancer (5).

These complications can reduce one's hope and quality of life (QOL) (6). In Baczewska et al.'s study (2019), more than $70 \%$ of cancer patients had moderate to below-average hope levels. Also, about $25 \%$ of patients were very frustrated (7). Many of the previous studies have reported poor and low QOL in cancer patients (8).

Hope is defined as believing in positive outcomes following adverse events in life and developing solutions to achieve goals (9). Hopelessness can reduce QOL. In contrast, increased hope empowers cancer patients to fight against adverse situations during diagnosis and treatments. This ability reduces the 
symptoms and complications of cancer and ultimately improves the patient's QOL (10).

According to the World Health Organization (WHO), QOL is defined as a person's perceptions of their life, values, goals, standards, and interests (11). Like the concept of hope, it also addresses the mental, social, and psychological dimensions of cancer patients.

Since hopelessness and decreased quality of life can lead to reduced acceptance and tolerance of cancer complications, therapeutic interventions are needed to increase the hope and QOL of cancer patients (12). Hope therapies, cognitive-behavioral therapies, and drug therapies with few side effects are among the methods used to increase the hope and QOL of cancer patients, each of which takes a relatively long time $(13,14)$. Dignity therapy (DT) is also among possible interventions. Dignity therapy (DT) is a short-term intervention (three to four 60-minute sessions) that can be implemented by nurses in a patient's home or in health centers to improve the hope and quality of life of chronic patients (14).

Dignity refers to one's perception of their value and competence. In DT, concepts such as respect for the patient's rights and privacy, independence, authority, and communication with the patient's family and staff are considered (15). This intervention encourages patients to talk about and comment on the most important issues in life, their desires, and their feelings (16). During DT sessions, the patient is asked questions such as the following: "What experiences have you had in life?" What expectations do you have for your closest person?" "What makes you feel proud?" "What are your wishes and demands from those around you?" "What is the most important achievement of your life?" The patient's responses and wishes are relayed to their loved ones via a generative document to provide the patient as much as possible $(15,16)$.

In nursing, respect and dignity of patients are considered essential parts of care regardless of the patient's ethnicity or their social and economic status (17). Researchers in the field of psycho-oncology believe that paying attention to patients' wishes and providing opportunities for them to meet their physical, mental, and psychological needs, lead to improved status (15-17).

Various studies have investigated the effect of DT on patients' hope, with conflicting results reported. Vaghee et al.'s study (2012) on hemodialysis patients and Montross et al.'s study (2015) on cancer patients showed that dignity therapy leads to increased hope in mentioned patients $(\mathbf{1 8}, \mathbf{1 9})$. However, Bentley et al. 's study (2014) on caregivers of patients with motor neuron disease and Aoun et al.'s study (2015) on motor neuron patients and their caregivers showed that DT did not significantly increase hope $(20,21)$.

The effects of DT on quality of life also differ. Chochinov et al. $(2005,2011)$ studied near-death patients, Rudilla et al. hospitalized patients (2016), and Johnston et al. in dementia patients (2016) and all reported that DT improved the quality of life $(14,22-$ 24). However, the results of a study by Aoun et al. (2015) in patients with neuron-motor diseases and their caregivers, and Vuksanovic et al. (2017) in patients with severe diseases showed that DT does not affect the quality of life $(21,25)$.

As mentioned above, results on the effect of DT on the hope and QOL of patients with chronic conditions, including cancer patients, are inconsistent. Perhaps DT leads to different results in diverse cultural contexts (26). Finally, no research has been undertaken to investigate the impact of dignity therapy on the hope and quality of life of cancer patients in the context of Iranian culture. Therefore, the present study was designed to determine the effect of dignity therapy on hope and quality of life in cancer patients.

\section{Materials and Methods}

The present study is a randomized controlled clinical trial (IRCT20190604043820N1) conducted at Iran Mehr Hospital in Birjand in 2019. The study population consisted of all cancer patients in this hospital. The sample size was calculated according to the study by Vaghee et al. (2013) with $80 \%$ power and an attrition rate of $10 \%$ (27). These values were selected using consensus sampling according to the inclusion criteria.

$$
\mathrm{n}=\frac{\left(\mathrm{z}_{1-\frac{\alpha}{2}}+\mathrm{Z}_{1-\beta}\right)^{2}\left(\delta_{1}^{2}+\delta_{2}^{2}\right)}{\left(\mu_{1}-\mu_{2}\right)^{2}}=\frac{(1.96+0.84)^{2}(10.24+5.10)}{(32.2-34.1)^{2}}=34
$$

Inclusion criteria included a willingness to participate in the study, an age of 18 years or older, having elapsed six months since cancer diagnosis, elementary education at least, and awareness of cancer, and a score of 21 or more on a mini-mental state examination (MMSE). Exclusion criteria included unwillingness to participate in research, lack of cooperation of the patient's trusted party (family member or friend) to receive the generative document, psychosis, and a desire to migrate or travel during the intervention.

All participants completed the demographic information form, the Herth-Hope scale, and the quality of life questionnaire. Afterward, 76 participants were randomly assigned to the intervention group $(n=38)$ and the control group $(n=38)$.

\section{Data Collection}

Demographic questionnaires_-including age, gender, employment status, educational level, marital status, type of cancer, type of treatment, and time of 
cancer diagnosis, MMSE (1975), Hope Herth Index (HHI, 1991), and quality of life questionnaire for cancer patients (EORTC QLQ-C30) (1988) were used to collect data.

The participants' mental health and cognitive approval were assessed using the MMSE (1975). The MMSE consists of 11 questions and five domains (orientation, attention, computation, memory, and linguistic and motor skills). The minimum and maximum scores in MMSE are 0 to 30, respectively. A score of 21 or higher allowed participants to enter the study. The Cronbach's alpha for this tool was previously reported as 0.89 (28). In the present study, Cronbach's alpha was 0.86 .

The HHI consisted of 12 questions and three subscales of cognitive-temporal (Questions 1, 2, 6, and 11), affective-behavioral (Questions 4, 7, 10, and 12), and affinitive-contextual (Questions 3, 5, 8, 9). Responses to the HHI criterion terms are based on a 3point Likert scale $(1=$ Disagree, $2=$ Not sure, and $3=$ Agree). Questions 3 and 6 are scored in reverse. The total hope score is 12 to 36 points. Higher scores demonstrate a higher hope level. A score of 12 to 24 indicates a low level of hope, a score of 25 to 30 indicates a moderate level, and a score of 31 to 36 indicates a high level of hope. This tool was translated into Persian by Abdi et al. (2007), and its validity has been confirmed (10). In the present study, The Cronbach's alpha of the Herth-Hope questionnaire was 0.81 .

Assessment of quality of life of cancer patients was performed using EORTC QLQ-C30 scoring. The questionnaire consists of 30 statements that examine the quality of life of cancer patients in terms of function, symptoms, and general health dimensions. The functional dimension has five subscales and 16 items. Sub-scales include physical functioning (Items 1, 2, 3, 4, and 5), role-playing (Items 6 and 7), emotional functioning (Items 20 and 25), cognitive functioning (Items 21, 22, 23, and 24), and social functioning (Items 26, 27, and 9). The symptom dimension has nine subscales and 12 terms. Symptoms include fatigue (Items 10, 12, and 18), nausea and vomiting (Items 14 and 15), pain (Items 9 and 19), shortness of breath (Item 8), sleep disturbances (Item 11), decreased appetite (Item 13), constipation (Item 16), diarrhea (Item 17), and economic problems caused by the disease (Item 28). Finally, the general health dimension includes Items 29 and 30. The general health dimension uses a 7-point Likert scale, with possible responses ranging from very poor (0) to excellent (7); all other dimensions (functional and symptoms) use a 4-point Likert scale: Not at all (1), A little (2), Quite a bit (3), Very much (4). The minimum and maximum scores obtained in each dimension are 0 to 100 . In terms of symptoms, lower scores mean less adverse symptoms, but in other aspects, higher scores mean better and more desirable conditions. The reliability and validity of this questionnaire have been confirmed in various studies and have been used in clinical trials. Cronbach's alpha coefficient for this questionnaire was reported to be favorable. In other studies, the Cronbach's alpha of the quality of life questionnaire for cancer patients was reported to be greater than 0.70 (29). Cronbach's alpha was 0.79 in the present study. In the present study, the overall scores of hope and quality of life were reported.

\section{Ethical Considerations:}

This research was approved by the Ethics Committee of Birjand University of Medical Sciences (IR.BUMS.REC.1397.267). The researcher explained the purpose of the study and methodology to all participants. After signing an informed consent, participants were told that they could withdraw from the study at any stage without paying any fees. They were assured of the confidentiality of the collected data.

\section{Intervention:}

Before the DT sessions began, the corresponding author was trained for 70 hours under the supervision of a psychologist who had the necessary expertise in the field of dignity therapy. After confirming and receiving the certificate, the researcher began the intervention.

In the first step of intervention (two weeks before the beginning of the sessions), the participants were given a complete schedule of days, attendance hours, meeting locations, and dignity therapy protocol questions in writing.

The basis of dignity therapy includes the preservation of independence, respect for the privacy of patients regardless of their social status and ethnicity, and non-judgment of patients. All of the above were considered and observed by the corresponding author. Only the corresponding author and the patient were present for the meetings. During each session, the conversations were recorded using a digital audio device. In each session, the researcher asked the patient questions about the dignity therapy protocol, and the participant answered. By using openended questions like "If your life is like a movie, which part is more important to you?" and "At what point in life did you feel alive?" the corresponding author easily facilitated patients' responses to protocol questions and their recall of memories. At the end of each session, the patient was given the opportunity to express what he or she thought was important but overlooked. The entire DT protocol was performed for each participant over three or four sessions of 30 to 45 minutes. The method of performing the intervention was taken from the reference book entitled "Dignity Therapy Final Words for Final Days" written by Chochinov (14) (Table 1).

At the end of all sessions, the recorded speeches of the participants were written with respect to integrity. Words, phrases, and sections unrelated to the protocol questions were deleted. Sentences containing inappropriate, offensive, or unpleasant words were 
corrected and rewritten with the direct participation of the patients. In the last step of the writing process, the most important phrase or sentence in the interview text from the participant's point of view was included at the end of the written document. The generated document - which contained the important memories, wishes, aspirations, and concerns of the patient-was provided to the patient for final correction and approval. If there was a discrepancy in the text of the re-document, the amendments were made under the direct supervision of the participant. In the next step, the patient's trustee (a friend or family member) was invited to the research environment and was given a description of the generative document intervention process. With the coordination and consent of the patient, the recipient could provide the generative document to other family members so that they could become familiar with the patient's thoughts, wishes, desires, past, and even meet the patient's needs as much as possible. The control group received routine care provided by the treatment center. Four weeks after the end of the intervention, participants from the two groups completed the HHI and the EORTC QLQ-C3.

Data were analyzed using SPSS 16 (SPSS Inc., Chicago, IL., USA). Descriptive statistics (frequency, percentage, mean, standard deviation) and analytical statistics were considered. Data were analyzed using the Kolmogorov-Smirnov test. A Wilcoxon test was used to compare the mean scores of hope between the two groups, and a Mann-Whitney test was used to compare the mean scores of hope within each group. A paired t-test was used to compare the mean scores of quality of life before and after the intervention within groups. An independent t-test was used to compare the quality of life scores between the two groups. The significance level was set at $\mathrm{P}$-value $<0.05$.

\section{Results}

Of the 76 participants in the present study, three individuals from the intervention and three from the control group were excluded. Data from the remaining 70 patients (35 from each group) were analyzed (Figure 1).

There were 28 females in the intervention group and 27 females in the control group. The mean ages of the intervention and control groups were 47.14 \pm 9.82 and $47.89 \pm 9.62$ years, respectively. The intervention group had the highest frequency of elementary education, and the control group had the highest number of diploma degrees. In the intervention group, there was one unmarried person, and all participants in the control group were married. Twenty patients in the intervention group and 23 patients in the control group were housewives. The most common type of cancer in both groups was breast cancer. The most common type of treatment was chemotherapy in both groups. Considering demographics, there were no statistically significant differences between the two groups $(P>0.05)$ (Table 2).

The Mann-Whitney test results showed that there was no significant difference between the mean scores of hope between the intervention $(24.02 \pm 3.05)$ and the control groups $(25.06 \pm 4.12)$ before the intervention $(P=0.11)$. In the intervention group before the intervention, the mean score of hope was $24.02 \pm 3.05$, which increased to $26.88 \pm 2.90$ four weeks after the completion of the intervention. This increase was statistically significant $(P<0.001)$. In the control group, there was no significant difference between the mean scores of hope before and after the intervention $(P=0.07)$. The results of the MannWhitney test showed that after the intervention, the mean scores of hope in the intervention group were significantly higher than those of the control group $(P=0.03)$ (Table 3). The mean change in the hope scores in the intervention group was $2.86 \pm 2.92$. The mean scores of hope in the intervention group were significantly higher than those of the control group $(P<0.001)$ (Table 4).

Independent t-test results showed no significant differences between the mean scores of quality of life between the intervention group $(51.93 \pm 15.55)$ and the control group $(48.03 \pm 12.54)(P=0.45)$. The paired ttest results showed that in the intervention group, the mean scores of quality of life after the intervention $(69.61 \pm 12.71)$ were significantly increased when compared with before the intervention $(51.93 \pm 15.50)$ $(P<0.001)$. In the control group, there was no significant difference between pre- and postintervention scores $(P=0.09)$. The independent t-test showed that four weeks after the intervention, the quality of life scores in the intervention group were significantly higher than those of the control group $(P<0.001)$. The mean score of quality of life in the intervention group was (17.68 \pm 12.37$)$ (Table 3). Mean changes in quality of life in the intervention group were significantly higher than in the control group $(P<0.001)$ (Table 4).

\section{Table 1. The Dignity Therapy Question Protocol}

\section{The Dignity Therapy Questions}

1) Tell me a little about your life history; particularly the parts that you either remember most or think are the most important.

(2) When did you feel most alive? 


\section{The Dignity Therapy Questions}

(3) Are there specific things that you would want your family to know about you,

(4) Are there particular things you would want them to remember?

(5) What are the most important roles you have played in life (family roles, vocational roles, communityservice roles, etc.)?

(6) Why were they important to you, and what do you think you accomplished in those roles?

(7) What are your most important accomplishments, and what do you feel most proud of?

(8) Are there particular things that you feel still need to be said to your loved ones (9) or things that you would want to take the time to say once again?

(10) What are your hopes and dreams for your loved ones?

(11) What have you learned about life that you would want to pass along to others? (12) What advice or words of guidance would you wish to pass along to your (son, daughter, husband, wife, parents, other[s])?

(13) Are there words or perhaps even instructions that you would like to offer your family to help prepare them for the future?

(14) In creating this permanent record, are there other things that you would like include?

*Numbering is only to ensure traceability of methodology and results for the reader

Table 2. Comparison of demographic characteristics of patients in the intervention and control groups

\begin{tabular}{|c|c|c|c|c|c|}
\hline \multicolumn{2}{|c|}{$\begin{array}{c}\text { Groups } \\
\text { Variables }\end{array}$} & $\begin{array}{l}\text { Intervention } \\
\text { Frequency (\%) }\end{array}$ & $\begin{array}{c}\text { Control } \\
\text { Frequency }(\%)\end{array}$ & $x^{2}$ & P-value \\
\hline \multicolumn{2}{|c|}{ Age } & $47.14 \pm 9.82$ & $47.89 \pm 9.62$ & 0.57 & 0.92 \\
\hline \multirow{2}{*}{ Gender } & Female & $28(80)$ & $27(77.1)$ & \multirow{2}{*}{0.09} & \multirow{2}{*}{0.77} \\
\hline & Male & $7(20)$ & $8(22.9)$ & & \\
\hline \multirow{4}{*}{ Educational level } & elementary & $12(34.3)$ & $11(31.4)$ & \multirow{4}{*}{3.82} & \multirow{4}{*}{0.28} \\
\hline & Middle & $4(11.4)$ & $8(22.9)$ & & \\
\hline & Diploma & $11(31.4)$ & $13(37.1)$ & & \\
\hline & Academic & $8(22.9)$ & $3(8.6)$ & & \\
\hline \multirow{2}{*}{ Marital status } & Single & $1(2.9)$ & $0(0)$ & \multirow{2}{*}{0.3} & \multirow{2}{*}{1.00} \\
\hline & Married & $34(97.1)$ & $35(100)$ & & \\
\hline \multirow{4}{*}{ Job status } & Employee & $7(20)$ & $3(8.6)$ & \multirow{4}{*}{2.35} & \multirow{4}{*}{0.50} \\
\hline & Retired & 4 (11.4) & $3(8.6)$ & & \\
\hline & housewife & $20(57.1)$ & $23(65.7)$ & & \\
\hline & Free & 4 (11.4) & $6(17.1)$ & & \\
\hline \multirow{4}{*}{ Type of cancer } & Lymphoma & $3(8.6)$ & $3(8.6)$ & \multirow{4}{*}{0.59} & \multirow{4}{*}{0.90} \\
\hline & Breast & $21(60)$ & $23(65.7)$ & & \\
\hline & Digestive cancer & $6(17.1)$ & $6(17.1)$ & & \\
\hline & Other & $5(14.3)$ & $3(8.6)$ & & \\
\hline \multirow{2}{*}{$\begin{array}{l}\text { Type of } \\
\text { treatment }\end{array}$} & Chemotherapy & $27(77.14)$ & $31(88.57)$ & \multirow{2}{*}{2.76} & \multirow{2}{*}{0.34} \\
\hline & Radiotherapy & $8(22.86)$ & $4(11.24)$ & & \\
\hline \multirow{5}{*}{$\begin{array}{l}\text { Time to diagnose } \\
\text { cancer }\end{array}$} & 6 to $8 \mathrm{M}$. ago & $9(25.71)$ & $7(20.00)$ & \multirow{5}{*}{2.05} & \multirow{5}{*}{0.48} \\
\hline & 9 to $11 \mathrm{M}$. ago & $5(14.28)$ & $13(37.14)$ & & \\
\hline & 12 to $14 \mathrm{M}$. ago & $8(22.85)$ & $10(28.57)$ & & \\
\hline & 15 to $17 \mathrm{M}$. ago & $10(28.57)$ & $2(5.71)$ & & \\
\hline & More than $17 \mathrm{M}$. ago & $3(8.59)$ & $3(8.58)$ & & \\
\hline
\end{tabular}


Table 3. Comparison of mean scores of hope and quality of life before and 4 weeks after intervention in two groups

\begin{tabular}{|c|c|c|c|c|}
\hline Variable & Time & Groups & & P-value ${ }^{b}$ \\
\hline & & $\begin{array}{l}\text { Intervention } \\
\text { Mean } \pm \text { S.D }\end{array}$ & $\begin{array}{l}\text { Control } \\
\text { Mean } \pm \text { S.D }\end{array}$ & \\
\hline \multirow{4}{*}{ Hope } & Before & $3.05 \pm 24.02$ & $4.12 \pm 25.06$ & $0.11, \mathrm{t}=1.36$ \\
\hline & After & $2.90 \pm 26.88$ & $4.26 \pm 24.60$ & $0.03, \mathrm{t}=4.79$ \\
\hline & P-value ${ }^{a}$ & $<0.001, \quad z=5.69$ & $0.20, z=3.96$ & \\
\hline & & & & P-value ${ }^{d}$ \\
\hline \multirow{3}{*}{ Quality of life } & Before & $15.50 \pm 51.93$ & $12.54 \pm 48.03$ & $0.45, \mathrm{~T}=2.27$ \\
\hline & After & $12.71 \pm 69.61$ & $12.15 \pm 50.64$ & $<0.001, \mathrm{t}=5.31$ \\
\hline & $\mathrm{P}$-value ${ }^{\mathrm{c}}$ & $<0.001, \mathrm{t}=6.62$ & $0.09, \mathrm{t}=0.27$ & \\
\hline
\end{tabular}

Table 4. Comparison of mean changes in hope and quality of life scores before and 4 weeks after intervention in two groups.

\begin{tabular}{llll} 
& Intervention & Control & P-value $^{\mathrm{d}}$ \\
\hline Hope & $2.92 \pm 2.86$ & $4.17- \pm 0.46$ & $<0.001, \mathrm{t}=5.17$ \\
\hline Quality of life & $12.37 \pm 17.68$ & $12.30 \pm 2.61$ & $<0.001, \mathrm{t}=5.83$ \\
\hline
\end{tabular}

\footnotetext{
d Independent samples t-test
}

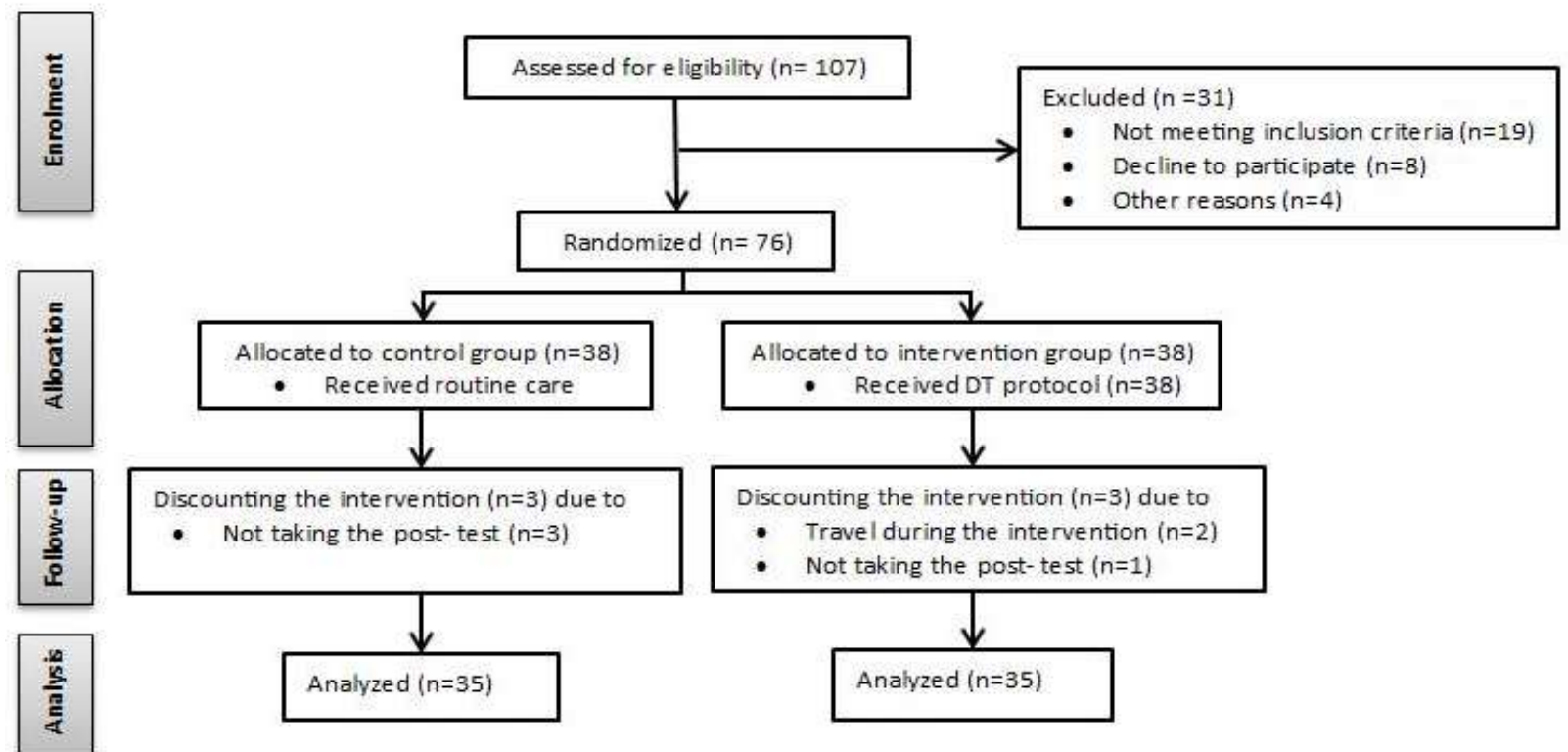

Figure 1. Consort flow diagram of study

\section{Discussion}

The results showed that four weeks after the intervention, the mean hope scores and mean changes of hope scores in the intervention group were significantly increased when compared to the control group. In other words, dignity therapy increased the hope of cancer patients.
Research by Vaghee et al. (2012) on the effect of dignity therapy on hope in hemodialysis patients is in line with the results of the present study (18). The results of Montross et al.'s (2015) study also confirm the findings of the present study (19). 
One of the possible mechanisms of the effect of dignity therapy on hope among cancer patients is that it creates a sense of meaning and purpose in life. The generative document containing the patients' feelings, interests, and desires led them to think and make decisions about their future lives. This treatment also enhances self-esteem, self-worth, and self-efficacy in patients by reminding them of their happy and lively days and their previous successes. Increasing one's confidence in his/her abilities leads to improved selfesteem and dignity (30). Increasing perceived dignity and self-worth is a driving force for increased effort and the use of problem-solving techniques to achieve goals. It has been suggested that applying problemsolving and problem-focused coping styles can lead to increased hope (31). It may also have helped patients find ways to improve their emotional relationships with loved ones. As a result of this intervention, relatives and loved ones of the patient appear to have a better understanding of the patients' interests, wishes, and needs. The patient's loved ones and relatives who were the recipients of the generative document paid attention to the patient's wishes and requests with empathy, attention, interest, and sensitivity.

However, the results of Bentley et al.'s (2014) study on the feasibility, acceptance, and effectiveness of dignity therapy for family caregivers of people with neuron-motor disorders showed that dignity therapy did not have a significant effect on improving the hope of these family caregivers (20). In a study aimed at determining the effect of dignity therapy on neuronmotor disorder patients and their caregivers, Aoun et al. (2015) found that dignity therapy did not affect participants' hope (21). The reasons for the inconsistency between the results of the mentioned research and the present study include the differences in target groups, duration of intervention, and outcome measurement time, as well as differences in the tools used to measure hope. Most participants in the study by Bentley et al. and Aoun et al. were amyotrophic lateral sclerosis (ALS) patients. The physical changes and the onset of disabling symptoms are extremely severe and affect the entire nervous and motor systems of the patient in a short time (32). This can negatively affect hope for the future. Another reason for the inconsistency of the findings of this study with those of Bentley et al. is that in the present study, hope was measured four weeks after the end of the intervention. But in the study by Bentley et al., outcomes were measured one week after the intervention. In the present study, the patient's family and loved ones were more likely to have the opportunity to make the patient's wishes and aspirations come true after the end of intervention. However, in Bentley et al.'s study, patients' families had little time to help the patient achieve his or her goals.

The results showed that four weeks after the end of the intervention, the mean scores and changes in mean scores for QOL in the intervention group were significantly increased when compared with the control group. In other words, dignity therapy led to increased QOL for cancer patients. Research by Chochinov et al. (2005, 2011), which investigated the effect of dignity therapy on different aspects of near-death patients, showed that dignity therapy improves QOL (14,22). Research findings by Rudilla et al. (2016) comparing psychological counseling with dignity therapy in home care patients also indicated a favorable effect of dignity therapy on improving QOL (23). In addition, the results of Johnston et al.'s (2016) study that investigated the effect of dignity therapy on patients with dementia showed that this intervention improves QOL (24). The results of all the above-mentioned studies are in line with the findings of the present study.

Dignity therapy is based on two basic principles. The first is the patients' perception of themselves as human beings and the concept of self-worth, and the second is the active involvement of the patients' loved ones and associates in helping the patients achieve their goals and aspirations. In other words, an understanding of patients' condition and their wishes and requests can lead to social support. Social support is an important factor that has significant effects on the QOL of cancer patients (33). The availability of social support resources has favorable effects on cancer patients' well-being and health. Hodges et al. (2012) believed that social support increases the quality of life. Meanwhile, a lack of social support leads to ineffective coping strategies and the exacerbation of the patients' psychological distress (34), consequently reducing their QOL. Paying attention to the wishes, requests, and desires of patients - regardless of their disease, gender, or economic status-increases the hope of patients. Increasing hope empowers cancer patients to fight against adverse situations and plays an important role in improving life satisfaction (33). As hope and QOL are directly and intimately linked $(19,20)$, increasing hope for the future will lead cancer patients to take measures to improve their physical and mental health, thereby improving their QOL (35).

However, the results of Aoun et al.'s (2015) examination of the effect of dignity therapy on patients with neuron-motor disorders and their families showed that dignity therapy did not affect patients' QOL (21). The results of Vuksanovic et al.'s (2017) study on the effect of dignity therapy on near-death patients showed that despite patients' acceptance of this treatment, dignity therapy had no impact on QOL (emotional, physical, social, and functional aspects of patients) (25). In explaining and interpreting the inconsistency of the results of the present study with the findings of Aoun et al.'s and Vuksanovic et al.'s studies, we point to the difference in the type of disease of the participants. The target group in the present study was cancer patients. In Aoun et al.'s study, the participants were suffering from neuron-motor disorders. Another reason for the inconsistency is the difference in the scales used to measure the QOL, as well as the length of the interventions. It is recommended that future studies compare the impact of dignity therapy on QOL 
with other palliative care methods. It is also recommended that future works investigate the effect of dignity therapy on quality of life and the symptoms of physical and psychological distress in chronic patients such as those suffering from multiple sclerosis and physical disabilities.

\section{Conclusion}

Dignity therapy can be a useful intervention method for increasing hope and improving the quality of life of cancer patients.

\section{Study Limitations}

One of the limitations of the present study is the small sample size. Also, all participants were patients of a single health center.

\section{Acknowledgments}

The authors thank the vice-chancellor of research and technology at Birjand University of Medical Sciences for sponsoring this research project. We would also like to thank all the participants who took part in this study.

\section{Funding}

This research did not receive any specific grant from funding agencies in the public, commercial, or not-forprofit sectors.

\section{Conflict of Interest}

Authors declared no conflict of interest.

\section{References}

1. Siegel RL, Miller KD, Jemal A. Cancer statistics, 2019. CA: Cancer J Clin. 2019;69(1):7-34 [DOI:10.3322/caac.21551]

2. Mousavi SM, Gouya MM, Ramazani R, Davanlou M, Hajsadeghi N, Seddighi Z. Cancer incidence and mortality in Iran. Anna Oncol. 2009;20(3):55663. [DOI:10.1093/annonc/mdn642]

3. Guimaraes MD, Bitencourt AG, Marchiori E, Chojniak R, Gross JL, Kundra V. Imaging acute complications in cancer patients: what should be evaluated in the emergency setting? Cancer Image. 2014;14(1):18. [DOI:10.1186/1470-7330-14-18]

4. Ismail F, Mohamed A, Lim K. Systemic therapy of cancer. Kuala Lumpur: The Ministry of Health; 2011.
5. Kadan-Lottick NS, Vanderwerker LC, Block SD, Zhang B, Prigerson HG. Psychiatric disorders and mental health service use in patients with advanced cancer: a report from the coping with cancer study. Cancer: Interdiscip Int $\mathrm{J}$ Am Cancer Soc. 2005;104(12):2872-81. [DOI:10.1002/cncr.21532]

6. Oberoi DV, White VM, Seymour JF, et al. Distress and unmet needs during treatment and quality of life in early cancer survivorship: A longitudinal study of haematological cancer patients. Europ J Haematol. 2017;99(5):423-30. [DOI:10.1111/ejh.12941]

7. Baczewska B, Block B, Kropornicka B, et al. Hope in hospitalized patients with terminal cancer. Int J Environ Res Pub Health. 2019;16(20):3867. [DOI:10.3390/ijerph16203867]

8. Nayak MG, George A, Vidyasagar M, et al. Quality of life among cancer patients. Indian J Palliat Care. 2017;23(4):445. [DOI:10.4103/IJPC.IJPC 82_17]

9. Ebrahimi N, Sabaghian Z, Abolghasemi M. Investigating relationship of hope and academic success of college students. Quarter J Res Plan Higher Educat. 2011;17(2):1-16.

10. Abdi N, Taghdisi M, Naghdi S. The effects of hope promoting interventions on cancer patients. A case study in Sanandaj, Iran, in 2007. Armaghane Danesh. 2009;14(3):13-21.

11. Theofilou P. Quality of Life: Definition and Measurement. Europe J Psychol. 2013;9(1):150-61 [DOI:10.5964/ejop.v9i1.337]

12. Üstündağ S, Zencirci AD. Factors affecting the quality of life of cancer patients undergoing chemotherapy: A questionnaire study. Asia Pac J Oncol Nurs. 2015;2(1):17-25 [DOI:10.4103/23475625.152402]

13. Osborn RL, Demoncada AC, Feuerstein $M$. Psychosocial interventions for depression, anxiety, and quality of life in cancer survivors: metaanalyses. Int J Psychiat Med. 2006;36(1):13-34. [DOI:10.2190/EUFN-RV1K-Y3TR-FK0L]

14. Chochinov HM, Hack T, Hassard T, Kristjanson LJ, McClement S, Harlos M. Dignity therapy: a novel psychotherapeutic intervention for patients near the end of life. J Clin Oncol. 2005;23(24):5520-5. [DOI:10.1200/JCO.2005.08.391]

15. Griffin-Heslin VL. An analysis of the concept dignity. Accident and emergency nursing. 2005;13(4):251-7. [DOI:10.1016/j.aaen.2005.09.003]

16. Barnosky AR. Dignity Therapy: final words for final days. JAMA. 2012;307(23):2550-. [DOI:10.1001/jama.307.23.2550] 
17. Borhani F, Abbaszadeh A, Rabori RM. Facilitators and threats to the patient dignity in hospitalized patients with heart diseases: a qualitative study. Int J Comm Based Nurs Midwif. 2016;4(1):36.

18. Vaghee S, Javadi RA, Mazlom S, Davoudi N, Gharavi MM, Zare M. The effect of dignity therapy on hope level in patients with chronic renal failure undergoing hemodialysis. Life Sci J ACTA. 2012;9(4):3722-7.

19. Montross-Thomas LP, Irwin SA, Meier EA, et al. Enhancing legacy in palliative care: study protocol for a randomized controlled trial of Dignity Therapy focused on positive outcomes. BMC palliative care. 2015;14(1):44. [DOI:10.1186/s12904-015-0041-z]

20. Bentley B, O'Connor M, Breen LJ, Kane R. Feasibility, acceptability and potential effectiveness of dignity therapy for family carers of people with motor neurone disease. BMC palliative care. 2014;13(1):12. [DOI:10.1186/1472-684X13-12]

21. Aoun SM, Chochinov HM, Kristjanson LJ. Dignity therapy for people with motor neuron disease and their family caregivers: a feasibility study. J Palliat Med. 2015;18(1):31-7 [DOI:10.1089/jpm.2014.0213]

22. Chochinov HM, Kristjanson LJ, Breitbart W, et al. Effect of dignity therapy on distress and end-of-life experience in terminally ill patients: a randomised controlled trial. Lancet Oncol. 2011;12(8):753-62. [DOI:10.1016/S1470-2045(11)70153-X]

23. Rudilla D, Galiana L, Oliver A, Barreto P. Comparing counseling and dignity therapies in home care patients: A pilot study. Palliative \& Support Care. 2016;14(4):321-9. [DOI:10.1017/S1478951515001182]

24. Johnston B, Lawton S, McCaw C, et al. Living well with dementia: enhancing dignity and quality of life, using a novel intervention, Dignity Therapy. Int J Old People Nurs. 2016;11(2):107-20. [DOI:10.1111/opn.12103]

25. Vuksanovic D, Green HJ, Dyck M, Morrissey SA. Dignity therapy and life review for palliative care patients: a randomized controlled trial. J Pain and Symptom Manag. 2017;53(2):162-70. e1. [DOI:10.1016/j.jpainsymman.2016.09.005]

26. Akechi T, Akazawa T, Komori Y, et al. Dignity therapy: Preliminary cross-cultural findings regarding implementation among Japanese advanced cancer patients. Palliat Med. 2012;26(5):768. [DOI:10.1177/0269216312437214]
27. Vaghee S, Rabbani Javadi A, Mazlom S, Davoudi $\mathrm{N}$, Modares Gharav M. The effect of dignity therapy on human dignity in patients with chronic renal failure undergoing hemodialysis. Evidence Based Care. 2013;3(2):63-72.

28. Foroughan M, Jafari Z, Shirin Bayan P, Ghaem Magham Farahani Z, Rahgozar M. Validation of mini-mental state examination (MMSE) in the elderly population of Tehran. Adv Cog Sci. 2008;10(2):29-37.

29. Behzadipour S, Sepahmansour M, Keshavarzi F, Farzad V, Naziri G, Zamanian S. Effectiveness of stress management based on cognitive-behavioral intervention on guality of life and coping styles for female with breast cancer. 2013.

30. Kantor A, Lipsitt L, Woodard CR, Groden J. How everyone on the Autism spectrum, young and old, can...: become resilient, be more optimistic, enjoy humor, be Kind, and increase self-efficacy-A Positive Psychology Approach: Jessica Kingsley Publishers; 2011.

31. Zhang J, Wei G, Ping W, Wu Z-h. Relationships among hope, coping style and social support for breast cancer patients. Chinese Med J. 2010;123(17):2331-5.

32. Pagnini F, Rossi G, Lunetta C, et al. Burden, depression, and anxiety in caregivers of people with amyotrophic lateral sclerosis. Psychol, Health \& Med. 2010;15(6):685-93. [DOI:10.1080/13548506.2010.507773]

33. Li M-Y, Yang Y-L, Liu L, Wang L. Effects of social support, hope and resilience on quality of life among Chinese bladder cancer patients: a crosssectional study. Health Qual life Outcomes. 2016;14(1):73. [DOI:10.1186/s12955-016-0481-z]

34. Hodges K, Winstanley S. Effects of optimism, social support, fighting spirit, cancer worry and internal health locus of control on positive affect in cancer survivors: a path analysis. Stress and Health. 2012;28(5):408-15. [DOI:10.1002/smi.2471]

35. Rustøen T, Wiklund I, Hanestad BR, Moum T. Nursing intervention to increase hope and quality of life in newly diagnosed cancer patients. Cancer Nurs. 1998;21(4):235-45. [DOI:10.1097/00002820-199808000-00003] 


\section{How to Cite This Article:}

Karimi Z, Mehrpooya N, Vagharseyyedin S A, Rahimi H. Dignity Therapy Improves Hope and Quality of Life in Cancer Patients: A Randomized Clinical Trial. J Adv Med Biomed Res. 2020; 28 (128) :156-165

\section{Download citation:}

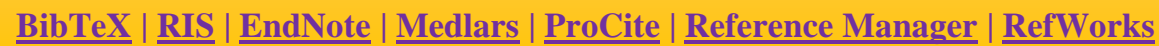

\section{Send citation to:}

Mendeley 2 Zotero Orefworka RefWorks 